\title{
Radiación fotosintéticamente activa evaluada en la ciudad de Zacatecas
}

\section{Photosynthetically active radiation evaluated in Zacatecas city}

ESCOBEDO-SÁNCHEZ, Mónica Montserrat†’, CONEJO-FLORES, Ricardo’, DURÓN-TORRES,

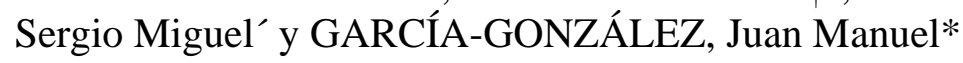

'Universidad Autónoma de Zacatecas. Unidad Académica de Ciencias Químicas, Programa Académico de Ingeniería Química, Campus UAZ Siglo XXI. Carretera Zacatecas - Guadalajara Km. 6, Ejido la Escondida, 98160 Zacatecas, Zac. México.

ID $1^{\mathrm{er}}$ Autor: Mónica Montserrat, Escobedo-Sánchez / ORC ID: 0000-0002-5300-7690, CVU CONACYT ID: 1014383

ID $1^{\mathrm{er}}$ Coautor: Ricardo, Conejo-Flores / ORC ID: 0000-0002-8513-1821, CVU CONACYT ID: 518968

ID $2^{\mathrm{do}}$ Coautor: Sergio Miguel, Durón-Torres / ORC ID: 0000-0003-2261-1681, CVU CONACYT ID: 5505

ID $3^{\text {er }}$ Coautor: Juan Manuel, García-González / ORC ID: 0000-0001-7259-5021, CVU CONACYT ID: 346241

DOI: $10.35429 /$ JCPE.2020.22.7.1.11

Recibido 10 de Enero, 2020; Aceptado 30 de Junio, 2020

\begin{abstract}
Resumen
La presente investigación se encuentra relacionada con uno de los procesos más importantes para el desarrollo de la vida en la Tierra; la fotosíntesis, proceso indispensable en el ciclo y desarrollo de los seres vivos, centrado en la radiación solar que le es útil a las plantas para llevar a cabo este proceso, la Radiación Fotosintéticamente Activa (PAR). El objetivo de este trabajo es generar información de la PAR a través de una base de datos para colaborar en la toma de decisiones de los agricultores del área. Para tal fin, se utilizó un sensor quantum instalado en el edificio 6 del Campus UAZ Siglo XXI. De acuerdo a Abal (2013) en la planificación agropecuaria y productiva, es muy importante contar con un conocimiento detallado de la radiación solar incidente en la superficie terrestre (Abal y Durañona, 2013). Al recopilar, tratar y analizar los datos, se encontró que la PAR promedio diaria es de $819.52 \mu \mathrm{mol}$ de fotones m-2 s-1 (179.47 Wm-2), si se toman en cuenta sólo las horas sol. Se puede concluir que de acuerdo con la PAR recibida en la región de evaluación y al tipo de nutrientes de la tierra, se pueden buscar otras alternativas de cultivos a los empleados tradicionalmente.
\end{abstract}

Sensor quantum, Radiación solar, Fotosíntesis

\begin{abstract}
The present investigation is related to one of the most important processes for the development of life on Earth; photosynthesis, an essential process in the cycle and development of living beings, centered on solar radiation that is useful for plants to carry out this process, Photosynthetically Active Radiation (PAR). The objective of this work is to generate information on the PAR through a database to collaborate in the decision-making of farmers in the area. For this purpose, a quantum sensor installed in building 6 of the UAZ Siglo XXI Campus was used. According to Abal (2013), in agricultural and production planning, it is especially important to have a detailed knowledge of incident solar radiation on the earth's surface (Abal and Durañona, 2013). When collecting, treating and analyzing the data, it was found that the daily average PAR is $819.52 \mu \mathrm{mol}$ of photons $\mathrm{m}-2 \mathrm{~s}-1(179.47 \mathrm{~W} \mathrm{~m}-2)$, if only the sunny hours are taken into account. It can be concluded that according to the PAR received in the evaluation region and the type of nutrients in the soil, other crop alternatives to those traditionally used can be sought.
\end{abstract}

Quantum sensor, Photosynthesis, Solar radiation

Citación: ESCOBEDO-SÁNCHEZ, Mónica Montserrat, CONEJO-FLORES, Ricardo, DURÓN-TORRES, Sergio Miguel y GARCÍA-GONZÁLEZ, Juan Manuel. Radiación fotosintéticamente activa evaluada en la ciudad de Zacatecas. Revista de Energía Química y Física. 2020. 7-22:1-11.

\footnotetext{
* Correspondencia al Autor (Correo Electrónico: jmgarcia@uaz.edu.mx)

$\dagger$ Investigador contribuyendo como primer autor
} 


\section{Introducción}

El Sol es una estrella que pertenece a nuestro Sistema Solar, se encuentra a una distancia media respecto a la Tierra de $1.5 \times 10^{11} \mathrm{~m}$, es la principal fuente de energía para la mayoría de los procesos biológicos conocidos que tienen lugar en nuestro planeta. Su energía procede de la transformación constante en su núcleo, átomos de hidrógeno en helio, este proceso libera gran cantidad de energía (Duffie et al, 2013).

La corona, es la superficie que se observa desde la tierra, la cual se estima que alcanza una temperatura promedio de 5,778 K (Caballinas, 2017). La radiación solar es la fuente primaria de energía para la vida sobre la Tierra, es esencial para la dinámica biológica de este planeta que alberga algo tan genuino como la vida (De las Rivas, 2000).

La fotosíntesis es el proceso en el que las plantas y otros organismos convierten la luz en energía química y luego en carbohidratos tales como el azúcar (Rojas, 2015). Se define como un único y complejo proceso fisicoquímico por el cual determinados organismos vivos autótrofos o fotosintéticos; es decir, organismos capaces de elaborar su propio alimento, absorben y utilizan la energía luminosa (De Las Rivas, 2000) para sintetizar compuestos orgánicos (Pérez, 2009).

La radiación fotosintéticamente activa o PAR puede ser definida como una fracción del espectro solar que está comprendida entre los $400 \mathrm{~nm}$ y los $700 \mathrm{~nm}$, que se encuentra en la zona visible del espectro, esta radiación es absorbida por las plantas, almacenada y transformada a través de sus sistemas fotosintéticos (Durán et al. 2015).

La radiación PAR es la fuente de energía para la fotosíntesis, que generalmente se estima como una fracción constante de la radiación solar global, frecuentemente se encuentra entre $35 \%$ a $50 \%$, dependiendo del tiempo y las condiciones climáticas (Durán et al. 2015).

La radiación PAR controla la fotosíntesis no sólo mediante su intensidad, sino también con la disponibilidad de longitudes de onda presentes y con el tiempo de exposición o fotoperiodo (Sager et al., 1997).
En el año 2014, la Secretaría de Energía del Gobierno Federal (SENER) y el Consejo Nacional de Ciencia y Tecnología (CONACYT), impulsaron el proyecto de creación del Centro Mexicano de Innovación en Energía Solar (CEMIESOL), el cual quedó a cargo del Instituto de Energías Renovables de la UNAM.

Entre los diversos subproyectos para el desarrollo de las tecnologías solares, se aprobó la creación del Inventario Nacional de Energía Solar, bajo la responsabilidad del Observatorio de Radiación Solar.

Esto permitió la instalación de la primera red solarimétrica de referencia a nivel nacional. La cual consta de 10 estaciones solarimétricas distribuidas en todo el territorio nacional, de acuerdo a una regionalización realizada a través de un método estadístico multivariado de análisis (método ISODATA).

La función primordial del Servicio Solarimétrico Mexicano es generar información precisa del mayor número de sitios en nuestro país y del mayor número de componentes de la radiación solar, a través de su red de estaciones solarimétricas.

Al mismo tiempo que genera, también recopila y valida información de otras redes y/o estaciones solarimétricas, que cumplen con las diferentes características establecidas por la Organización Meteorológica Mundial para su óptimo funcionamiento, las cuales van desde el emplazamiento del sitio, hasta el tipo de instrumentos y el mantenimiento de estos (Geofísica.unam.mx. 2019).

El objetivo de este trabajo es generar información de la PAR a través de una base de datos para colaborar en la toma de decisiones de los agricultores del área, ya que la siembra ha sido la tradicional dirigida al maíz y al frijol desde hace muchos años.

El reto en la región es contar con esa base de datos, aunada a algunos datos climatológicos como son la humedad y la temperatura del medio ambiente, así como, algunas características de la composición de la tierra de cultivo, lo cual coadyuve a otro tipo de cultivo. 


\section{La radiación solar}

La radiación solar es la energía electromagnética que fluye del Sol en forma natural (Caballinas, 2017), propagándose en ondas electromagnéticas en todas direcciones a través del espacio, viajan a la velocidad de la luz $(300,000 \mathrm{~km} / \mathrm{s})$ y no requieren algún medio de propagación y son de diferentes frecuencias comprendidas entre las regiones del visible, infrarrojo y UV, del espectro electromagnético.

La radiación recibida es la principal fuente de energía para la mayoría de los procesos que ocurren en nuestro planeta como los biológicos, además de determinar la dinámica de los procesos de la atmósfera y el clima (Benavides et al., 2017). La energía es tan abundante que puede aprovecharse para satisfacer las necesidades energéticas del hombre (Caballinas, 2017).

Es una energía sin costo, pero llega a la superficie en forma con gran variabilidad temporal, ahí se encuentra el desafío de la ingeniería para concentrarla, almacenarla y aprovecharla eficientemente a menor costo que otras fuentes de energía (Abalet et al., 2013).

La radiación electromagnética puede describirse también como fotones. (Resnick et al., 1997). Einstein propuso la noción de fotón (Resnick et al., 1997), planteó que la energía radiante es una corriente de diminutos paquetes de energía, llamados fotones. Extendiendo la teoría cuántica de Planck, Einstein dedujo que cada fotón contenía una energía proporcional a la frecuencia de la luz, representado por la ecuación 1.

Energía de fotón $=E=h v$

Donde: $v$ es la frecuencia de la radiación y $h$ es la constante de Planck, (Brown et al., 2004).

La radiación emitida por el Sol, juntamente con las condiciones geométricas con respecto a la Tierra, dan como resultado que, la radiación solar incidente sobre la superficie de la atmósfera, en una superficie perpendicular con una distancia media, es una cantidad casi constante y se denomina constante solar, Gsc, $\mathrm{G}_{\mathrm{SC}}=1361 \mathrm{~W} \cdot \mathrm{m}^{-2}$ (Duffie et al., 2013).

\section{El espectro electromagnético}

Las ondas electromagnéticas pueden tener diferentes longitudes de onda, el conjunto de todas las longitudes de onda se denomina espectro electromagnético. El conjunto de las longitudes de onda emitidas por el Sol se denomina espectro solar. El espectro electromagnético, (Figura 1), incluye una amplia gama de tipos de radiación emanada de diversas fuentes, y se distinguen únicamente por la longitud de onda o su frecuencia. Los nombres asignados a las regiones del espectro tienen que ver con la forma en que se producen u observan, no hay límites bien definidos entre una y otra categoría (Resnick et. al.,1997).

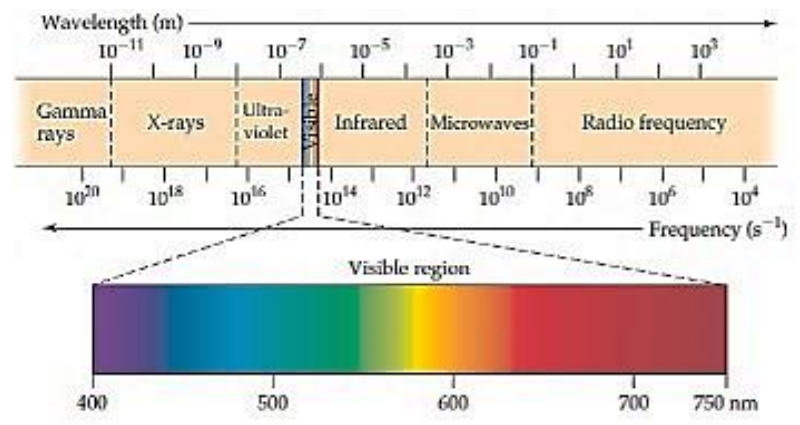

Figura 1 Espectro electromagnético

Fuente: Química la ciencia central (Brown et al., 2004).

La luz es la región visible del espectro y la más conocida puesto que los ojos cuentan con receptores adaptados sensibles a la radiación electromagnética. La distribución espectral de la radiación solar abarca todo el espectro electromagnético, pero la radiación más intensa se encuentra en la región que definimos como visible, que fluctúa entre los $400 \mathrm{~nm}$ a $700 \mathrm{~nm}$ (Resnick et al.,1997). Es conveniente considerar la radiación de Onda Corta con longitud de onda en el rango de $0.3 \mu \mathrm{m}$ hasta $3 \mu \mathrm{m}$ (Duffie et al., 2013), y de Onda Larga que se originan en fuentes cercanas a temperaturas ambientales presentes en la Tierra y son mucho mayor a $3 \mu \mathrm{m}$ (Duffie et al., 2013).

\section{El sistema Sol-Tierra}

La Tierra gira alrededor del Sol describiendo una órbita elíptica. El Sol se encuentra en uno de los focos y la distancia promedio Tierra-Sol es de aproximadamente de 149.46 x $10^{6} \mathrm{~km}$ (aprox. $1.5 \times 10^{11} \mathrm{~m}$ valor llamado Unidad Astronómica, UA). 
La excentricidad de la órbita de la Tierra es de $1.7 \%$, en el perihelio es la menor distancia entre el Sol y la Tierra (ocurre en enero) y es de $\mathrm{R}=\mathrm{UA}(1-\mathrm{e})=0.983 \mathrm{UA}=147.5 \times 10^{6} \mathrm{~km}$; el Afelio es la mayor distancia entre la Tierra y el Sol (ocurre en julio) y es de R = UA $(1+\mathrm{e})=$ $1.017 \mathrm{UA}=152.6 \times 10^{6} \mathrm{~km}$. Una vuelta alrededor del Sol es un año ciclo y se completa en 365 días, este movimiento es denominado de traslación, (Benavides et al., 2017). A su vez la Tierra gira alrededor de su propio eje con una duración aproximada de 24 horas, y a este fenómeno se le conoce como movimiento de rotación provocando el día y la noche (Caballinas, 2017).

El eje de la Tierra se encuentra ligeramente desplazado (23 $\left.3^{\circ} 26^{\prime} 7^{\prime \prime}\right)$ con respecto al plano de la órbita. Este plano recibe el nombre de eclíptica. El movimiento de traslación en conjunto con la oblicuidad terrestre produce las estaciones del año (Caballinas, 2017). Debido al movimiento de rotación el ángulo con el que los rayos solares inciden en la superficie terrestre varía entre $-23.45^{\circ}$ con respecto al Ecuador, cuando es el Solsticio de invierno (22 de diciembre) y $+23.45^{\circ}$ en el Solsticio de verano (21 de junio), siendo el día más largo (Benavides et al., 2017).

Dos veces al año el ángulo de incidencia se alinea con el Ecuador, esto ocurre durante los Equinoccios, el de otoño (23 de septiembre) y el de primavera (21 de marzo) los días y noches en estas fechas duran lo mismo. Debido a la inclinación del eje de rotación de la Tierra con respecto a la eclíptica en un mismo punto de la superficie terrestre los rayos del Sol inciden con una inclinación diferente, por lo tanto, la cantidad de energía solar que se recibe varía considerablemente. Lo que explica que alrededor del mediodía es mucho mayor la energía recibida, que en horas cercanas al amanecer o a la puesta de Sol (Benavideset al, 2017).

\section{La atmósfera terrestre}

No sólo las condiciones astronómicas influyen en la radiación solar disponible para una determinada región de la Tierra, depende también de la trayectoria de los rayos solares a través de la atmósfera.
La atmósfera terrestre es una mezcla no homogénea de varios gases y aerosoles, forman una capa que rodea a la Tierra, los gases que la componen son principalmente $\mathrm{O}_{3}, \mathrm{O}_{2}, \mathrm{~N}_{2}, \mathrm{CO}_{2}$, entre otros en cantidades muy pequeñas (Abal et al, 2013). La atmósfera absorbe, refleja y dispersa selectivamente la radiación solar, estos son los principales factores que cambian el flujo que incide en una determinada región de la superficie terrestre, cuando esta viaja por las distintas capas de la atmósfera. La magnitud de estos fenómenos depende de la masa relativa de la atmósfera (Bonhomme, 1993). Debido a estos procesos, parte de la radiación es absorbida, parte reflejada al espacio y otra fracción es dispersada en la atmósfera, en términos generales se calcula que alrededor del $23 \%$ de la radiación solar que incide en la atmósfera terrestre es alterado (Caballinas, 2017).

\section{La radiación PAR}

La radiación fotosintéticamente activa o PAR puede ser definida como una fracción del espectro solar que está comprendida entre los $400 \mathrm{~nm}$ y los $700 \mathrm{~nm}$, que se encuentra en la zona visible del espectro, esta radiación es absorbida por las plantas, almacenada y transformada a través de sus sistemas fotosintéticos (Durán et al. 2015). La radiación PAR es la fuente de energía para la fotosíntesis, que generalmente se estima como una fracción constante de la radiación solar global, frecuentemente se encuentra entre $35 \%$ a $50 \%$, dependiendo del tiempo y las condiciones climáticas (Durán et al. 2015).

La radiación PAR controla la fotosíntesis no sólo mediante su intensidad, sino también con la disponibilidad de longitudes de onda presentes y con el tiempo de exposición o fotoperiodo (Sager et al., 1997). La radiación PAR puede ser expresada en términos de flujo de fotones o irradiancia solar $\left(\mathrm{Wm}^{-2}\right)$ (Righini et al., 2005).

La densidad de flujo de fotones fotosintéticos, $\mathrm{Q}_{\mathrm{p}} \mathrm{o} \mathrm{PPF}$ por sus siglas en inglés y se define como la densidad del flujo de fotones incidentes con longitud de onda entre $400 \mathrm{~nm}$ a los $700 \mathrm{~nm}$ por unidad de tiempo sobre una unidad de superficie (1 $\mu \mathrm{mol}$ fotón $\cdot \mathrm{m}^{-2} \mathrm{~s}^{-1}=$ $6.022 \times 10^{17}$ fotón $\left.\cdot \mathrm{m}^{-2} \mathrm{~s}^{-1}=1 \mu \mathrm{E} \cdot \mathrm{m}^{-2} \mathrm{~s}^{-1}\right)$ (Alados et al., 2000). 
Como la absorción de la radiación PAR está regulada por los pigmentos de los cloroplastos, los máximos del espectro de absorción coinciden con los máximos de absorción de la clorofila y los carotenoides: la clorofila y algunos pigmentos son los receptores de la radiación (Grossi, 2004).

El nivel de radiación adecuado para saturar los sistemas fotosintéticos de plantas con el sistema de captura de carbón C-3 es generalmente cerca de los $400 \mu \mathrm{mol}$ fotón $\cdot \mathrm{m}^{-2}$ $\mathrm{s}^{-1}$ cuando se expone en periodos de $16 \mathrm{~h}$ por día. Algunas plantas particularmente las que tienen un sistema fotosintético C-4 requieren al menos niveles de radiación de $500 \mu \mathrm{mol}$ fotón $\cdot \mathrm{m}^{-2} \mathrm{~s}^{-1} \mathrm{o}$ mucho mayores con un fotoperiodo de $16 \mathrm{~h}$ diarias para maximizar su crecimiento.

Las plantas de follaje de crecimiento lento, necesita radiación PAR de 10 a $50 \mu \mathrm{mol}$ foton $\cdot \mathrm{m}^{-2} \mathrm{~s}^{-1} \mathrm{y}$ un fotoperiodo de 8 horas, con niveles más altos estas plantas tienden a no crecer o a secarse. Dentro de los trabajos en la literatura, donde se pone de manifiesto la influencia de la PAR en el crecimiento de las plantas se pueden destacar los presentados por: Liu et al., 2020) en el cual; examinaron la dependencia de dos recursos naturales sobre el tamaño de las plantas individuales en el sotobosque y los micrositios interespaciales, el contenido de agua del suelo y la luz en la superficie del suelo (radiación fotosintéticamente activa [PAR]).

Se reconoce que la influencia de la PAR fue significativamente menor en el sotobosque que en los micrositios interespaciales, y esta disminuyó ligeramente con el aumento del volumen de arbustos (Liu et. al, 2020); El presentado por Alvarado et. al (2020), en su trabajo, muestran que la densidad de población de plantas es el factor que influye en el rendimiento de una especie.

$\mathrm{Al}$ ser óptima, proporciona una adecuada cobertura foliar y esto permite interceptar la radiación fotosintéticamente activa. Pero también, comentan que densidades altas puede originar pérdida de plantas y alteraciones en su composición morfológica y química (Alvarado et. al, 2020).
El trabajo presentado por Planas (2020), el cual evaluó el nivel de radiación fotosintéticamente activa óptimo para cuatro variedades de Lolium perenne $L$. utilizadas en el Estadio Ramón Sánchez-Pizjuán y en función de la temperatura $\left(18\right.$ y $\left.10^{\circ} \mathrm{C}\right)$. La determinación de estos óptimos permitiría maximizar la eficiencia de las lámparas de luz artificial con el fin de ahorrar energía sin una disminución de la calidad del césped (Planas, 2020).

Por último, el trabajo de Yang (2020), donde analizó la relación entre el espectro de una fuente de luz y el espectro de reflexión de las hojas de las plantas, con el patrón de radiación fotosintéticamente activa, de las plantas desde la perspectiva de la teoría espectral. Encontró que una lámpara fluorescente tiene una característica espectral continua y puede proporcionar la mayor parte de la energía espectral requerida para el crecimiento de la planta (Yang, 2020).

La PAR es usualmente medida de dos formas. La primera puede ser de mediciones directas con ayuda de instrumentos, (como se realizó en este trabajo, además de los referenciados por Palomo et al., (2020) en la literatura, que desde 2006 realizan la evaluación de la PAR en la Agencia Estatal de Meteorología Española (Palomo et. al, 2020), y en la segunda los valores pueden ser estimados indirectamente de otros datos. Sin embargo, en términos de mediciones no se han establecido redes globales. Por lo tanto, la determinación de datos de la radiación PAR no están disponibles para muchas aplicaciones, como resultado, algunos investigadores han producido varios modelos de estimación (Simao et al., 2015).

Para le medición directa de la PAR existen actualmente sensores cuánticos, como el LI-190SA fabricado por LI-COR y el PAR Lite, de la empresa KIPP \& ZONEN. En ambos casos se utiliza como sensor un fotodiodo de silicio, al que se modifica su respuesta y se le agrega un filtro para proporcionar la respuesta en el rango de longitudes de onda en los que la radiación es mejor aprovechada por las plantas. Para propósitos prácticos, el contenido energético de radiación fotosintéticamente útil se ha considerado frecuentemente entre $35 \%$ a $50 \%$ de la global, dependiendo del tiempo y las condiciones climáticas (Grossi, 2004). 
Los sensores cuánticos tienen algunos errores espectrales causados por las respuestas no ideales, errores coseno causados por la respuesta de un ángulo incidente no ideal y la degradación a largo plazo de la respuesta debida a la exposición a la lluvia y radiación (Tomoko et al., 2015). El error espectral se observa con la radiación con varios patrones espectrales, como la luz del cielo. La respuesta a estos casos no ideales hace que los sensores quantum generen diferentes valores, incluso si la radiación PAR real es el mismo para los diferentes patrones espectrales.

En la actualidad, muchas investigaciones han trabajado esto para productos específicos bajo condiciones específicas para evaluar sus errores espectrales, por ejemplo Pearcy (1989) simuló los errores espectrales para dos tipos de sensores (un GaAsP fotodiodo y un sensor quantum usando un fotodiodo de $\mathrm{Si}$ ) se utilizan sus curvas de respuesta espectral inherentes y dos tipos de curvas de radiación espectral observadas (en condiciones de cielo despejado en la parte baja del bisque tropical de Costa Rica).

El error en el coseno es otro problema serio, especialmente cuando las observaciones (mediciones) en la radiación viene de varias direcciones. Si los resultados se toman en cuenta el coseno del ángulo incidente, son cuestionables, el sensor quantum arroja diferentes valores incluso si el valor actual de la PAR es igual para diferentes ángulos del zenit solar, nublado y con presencia de aerosoles.

En combinación del error coseno y el error espectral puede influir en la respuesta del sensor quantum de una manera muy compleja, sin embargo, tal complejidad y la combinación de estos errores en los sensores quantum no ha sido investigada. Otro factor significante de la degradación a la larga de la sensibilidad, especialmente en los monitoreos de las variaciones estacionales y anuales de la radiación PAR. Como se ha mencionado, la degradación del sensor es causada por la radiación UV y el vapor de agua; la velocidad y el grado de degradación depende de cómo el sensor es instalado y su exposición a la luz solar, lluvia y humedad (rocío).
Sin embargo, no se ha dado un estudio que presente un perfil claro de la degradación, el principal problema es la ausencia de un sistema de medición de la radiación PAR con la suficiente precisión y estabilidad que trabaje como un estándar de referencia para los sensores quantum para evaluar su precisión y estabilidad bajo las condiciones variables de luz (Tomoko et al., 2015).

Los métodos de estimación de la radiación PAR pueden ser clasificados en tres categorías: modelos físicos, modelos empíricos y modelos satelitales (Zhian et al., 2017).

Gueymard (1989) desarrolló dos modelos físicos para el cálculo de la PAR en condiciones de cielo despejado. Uno de los modelos fue diseñado específicamente por la banda espectral de la radiación PAR. Se usa la presión en la superficie, la cantidad de ozono, los coeficientes de turbiedad de Angstrom, la dispersión única de albedo, la superficie de albedo y el ángulo solar del zenit. Estos son los parámetros considerados para poder calcular la radiación PAR directamente.

Quin et al., (2012) desarrolló un modelo físico para la estimación diaria de radiación PAR con condiciones de cielo claro. El efecto de las nubes es considerado usando los datos de la duración de los rayos solares para calcular los valores de la radiación PAR diarios para las todas las condiciones del cielo. La ventaja de los modelos físicos, es que estos, normalmente son desarrollados con la relación física entre la PAR y las variables de las que depende, por lo tanto no hay restricción por el clima local o las condiciones geográficas (Zhian et al., 2017).

Un gran número de modelos empíricos han sido desarrollados para poder estimar la radiación PAR y han sido desarrollados con base en los datos de radiación PAR ya conocidos y la irradiancia global directa $\left(\mathrm{G}_{\mathrm{b}}\right)$. Estos modelos usualmente calculan el $\mathrm{Q}_{\mathrm{p}}$ o $\mathrm{Q}_{\mathrm{p}} / \mathrm{G}_{\mathrm{b}}$, como función de las medidas de radiación, índice de claridad y el ángulo con respecto al zenit solar. El índice de claridad es definido como una relación observada en la irradiancia global horizontal y la irradiancia global horizontal extraterrestre, que representa el efecto de la atmosfera en la radiación, se incluye la absorción por gases, nubes y aerosoles (Zhian et al., 2017). 
En general los modelos empíricos tienen como ventaja la simplicidad de forma y fácil uso. Estos también pueden producir estimaciones de PAR relativamente precisas en un área local, la desventaja de estos modelos es que están usualmente restringidos por las condiciones climáticas locales y por lo tanto la dependencia en los datos observados localmente. Otra limitación de estos métodos es que no pueden usados en áreas donde las variables necesarias no son disponibles (Zhian et al., 2017).

La radiación PAR también puede ser estimada mediante vía satélite. Rubio et al., (2005) usa las mediciones del satélite Meteosat para estimar la irradiancia global horizontal posteriormente se estima PAR usando la irradiancia global directa con un modelo empírico propuesto por Alados et al. (2000).

Zheng et al. (2008) propone un método de usar GOES visible Imágenes parrecuperar la radiación PAR. Las ventajas del método de Zeng es que en ambas superficies la reflectante y la atmosférica, los parámetros pueden ser simultáneamente derivados de las radiaciones observadas, lo anterior, sin requerir información de los parámetros de la superficie. La estimación de la radiación PAR mediante métodos satelitales tiene la ventaja de poder evaluar la radiación PAR en áreas remotas donde los datos para estimarla por otro método no están disponibles. Sin embargo, los métodos satelitales no pueden ser usados en la predicción numérica del clima (NWP) o el modelado de simulaciones de la radiación PAR en procesos de superficie terrestre.

Los métodos empíricos y satelitales tienen algunas restricciones en las aplicaciones, en cambio los modelos físicos están relativamente libres de restricciones y por lo tanto son preferidos (Zhian et al., 2017). La importancia de la predicción de variaciones temporales y espaciales de la intensidad de la PAR en los diferentes cultivos en donde se hacen los estudios es para poder así estimar mediante algunos modelos que se ha desarrollado la simulación el crecimiento y la productividad a partir de la PAR, no obstante, los valores de PAR utilizados en estos modelos se derivan de pequeños números de estudios empíricos debido a que esta variable no es evaluada rutinariamente (Duránet al., 2015).
Datos más detallados de la PAR ayudarán a la descripción y predicción de los modelos, muchas de las investigaciones de PAR están enfocados en los parámetros de cielo claro, muy pocos están enfocados en los efectos de las nubes sobre PAR (Simao et al. 2015).

\section{Metodología}

\section{Instrumento utilizado para el análisis}

El equipo utilizado en este análisis es un sensor cuántico de PAR "PQS 1 PAR Quantum Sensor", instalado en la Estación Solarimétrica Zacatecas_04 mostrada en la figura 2; este equipo consta de un fotodiodo de silicio de alta calidad y un filtro óptico de vidrio para crear una sensibilidad uniforme a la luz entre $400 \mathrm{~nm}$ y 700 $\mathrm{nm}$, su diseño permite ser instalado tanto en interiores como en exteriores.

Cuenta con un difusor proporcionando una excelente respuesta, el equipo hace una corrección automática al coseno, se brinda respuesta de coseno excelente que garantiza la precisión de las mediciones en luz difusa y cuando la luz solar proviene de un ángulo solar bajo. La brida del montaje incluye un nivel de burbuja.

\section{Características del sitio de medición}

Zacatecas está ubicado en la región centronorte del país cuenta con un clima templado subtropical de montaña. La temperatura media anual es de $17{ }^{\circ} \mathrm{C}$, la temperatura máxima promedio es alrededor de $30^{\circ} \mathrm{C}$ y se presenta en el mes de mayo, la temperatura mínima promedio es de $3{ }^{\circ} \mathrm{C}$ y se presenta en el mes de enero.

La precipitación media estatal es de 510 $\mathrm{mm}$ anuales, las lluvias se presentan en verano en los meses de junio a septiembre.

El clima seco y semiseco de la entidad es una limitante para la agricultura, ésta se practica de riego y temporal, existiendo en los principales cultivos de: maíz, avena, trigo, frijol, chile, sorgo, nopal y durazno (INEGI, 2018). 
Los datos fueron obtenidos de la Estación Solarimétrica Zacatecas_04 ubicada en el edificio E6 de la Unidad Académica de Ciencias Químicas en el Programa Académico de Ingeniería Química en el Campus UAZ Siglo XXI, ubicada en Carretera ZacatecasGuadalajara km. 6, Ejido "La Escondida", Zacatecas, Zac. con coordenadas Latitud (N) 22.7725 N Longitud (W) -102.6436 y una altitud de 2317 msnm (metros sobre el nivel del mar).

La Estación Solarimétrica Zacatecas forma parte de la Red Solarimétrica Mexicana del Servicio Solarimétrico Mexicano del Instituto de Geofísica de la Universidad Nacional Autónoma de México, esté es un sistema integral de medición, validación y publicación, con un mantenimiento permanente de equipo especializado para la medición de la radiación solar, que permite generar información confiable para la explotación de la energía solar para cualquier tipo de usuario.
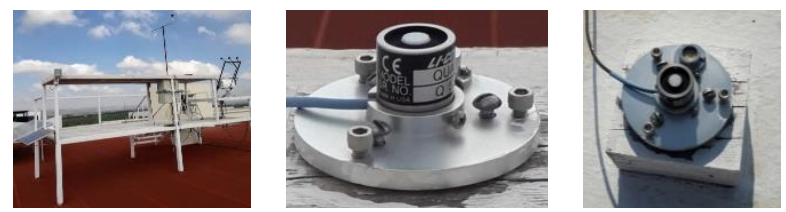

Figura 2 Sensor cuántico PAR y Estación Solarimétrica Zacatecas_04 ubicada en el edificio E6 de la UACQ del Programa Académico de Ingeniería Química del Campus Siglo XXI de la UAZ

\section{Procesamiento de resultados}

Los datos coleccionados de la Estación Zacatecas_04 por el sensor conectado a un adquisidor marca Campbell Scientific modelo CR3000, el cual mide cada 4 segundos todos los parámetros y almacena el promedio de cada minuto.

Los datos recopilados para este análisis comprenden un periodo de tiempo entre noviembre 2016 y noviembre 2017, fueron calculados en promedio por hora, promedio por día y promedio por mes.

Posteriormente se identificaron la hora en la que la mayoría de los valores promedios, el sensor registraba valores de PAR y la hora en que el sensor empezaba a registrar valores cercanos o iguales a cero, correspondientes al amanecer y el crepúsculo.
Ya con la hora aproximada tanto de amanecer y crepúsculo, se fijaron los datos de interés en los que la PAR incide en el sensor, con estos datos se obtuvieron gráficos con los promedios mensuales de las horas de interés. Se identificaron los días que registraron los promedios por hora PAR mayores y más bajos en cada mes, además del promedio por hora más alto y bajo registrado.

\section{Resultados}

Los datos de PAR obtenidos con el sensor Quantum están en unidades de densidad de flujo de fotones fotosintéticos, $\mu \mathrm{mol}$ de fotones $\mathrm{m}^{-2} \mathrm{~s}^{-}$ 1. El periodo del día en el que la PAR estaba presente se fijó entre las 6:00 h y las 19:00 h, correspondientes al amanecer y el crepúsculo respectivamente. En el Gráfico 1, se presentan los valores promedio obtenidos de la PAR para los meses de febrero y mayo de 2017, que corresponden a las estaciones de invierno y primavera; basados en la PAR registrada, se puede observar que en invierno los días son más cortos que en la primavera. Los promedios máximos para los meses comparados se alcanzan a las 13:00 h, con un valor de 1,681.64 $\frac{\mu \mathbf{m o l}}{\mathbf{s ~ m}^{2}}$, y a las 12:00 h con un valor promedio de la PAR de $1,962.40 \frac{\mu \mathbf{m o l}}{\mathbf{s ~ m}^{2}}$, respectivamente.

El mes de mayo de 2017 fue el mes en el cual se registró el valor promedio más alto de la PAR (1962.40 $\left.\frac{\mu \mathbf{m o l}}{\mathbf{s ~ m}^{2}}\right)$, y el mes de noviembre fue el más bajo de la PAR en el periodo evaluado (1272.69 $\left.\frac{\mu \mathbf{m o l}}{\mathbf{s ~ m}^{2}}\right)$. El día con el valor de la radiación PAR promedio por hora más alto registrado fue el 27 de junio 27 a las 12:00 h, con un valor de $2197.51 \mu \mathrm{mol} \mathrm{m} \mathrm{m}^{-2} \mathrm{~s}^{-1}$.

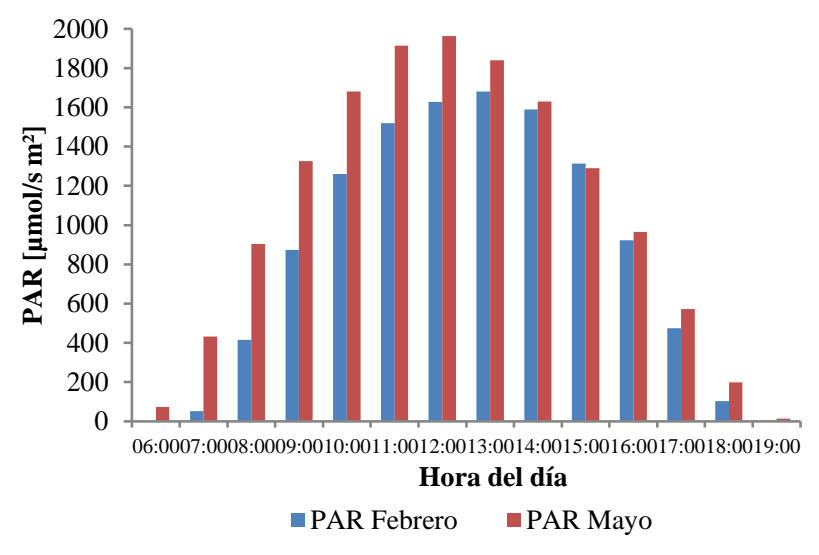

Gráfico 1 Comparación del promedio horario mensual de la PAR para febrero y mayo de 2017 
En el Gráfico 2, se presenta la PAR en el periodo de análisis comprendido de noviembre de 2016 hasta noviembre del 2017. Los meses correspondientes a los meses de otoño e invierno, se recibió menos energía PAR y los meses para las estaciones de primavera y verano son los que registraron los valores mayores de la PAR.

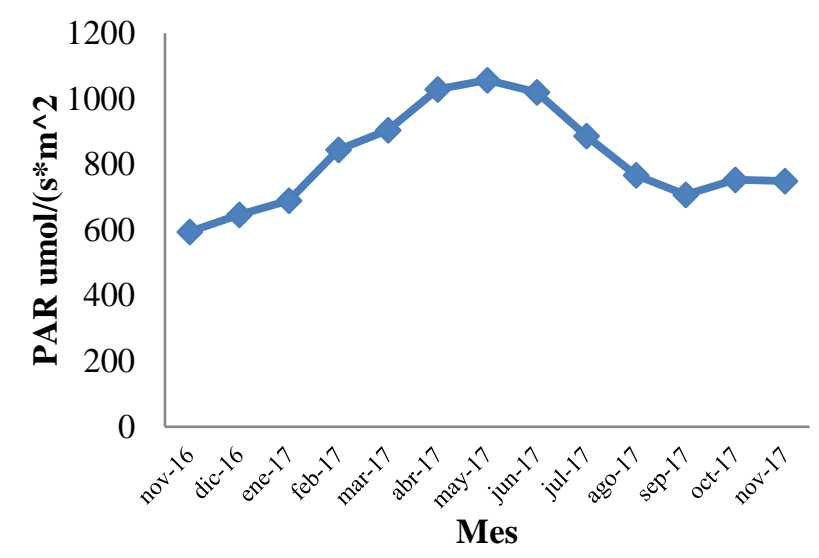

Gráfico 2 Promedios mensuales de la PAR en el periodo evaluado de noviembre de 2016 a noviembre de 2017

En el Gráfico 3, se presentan los registros promedio de PAR en el periodo de análisis. Se puede observar en él los típicos perfiles de la radiación PAR, afectados naturalmente por el desplazamiento del sol a lo largo del año con respecto a la Tierra, o a la inversa.

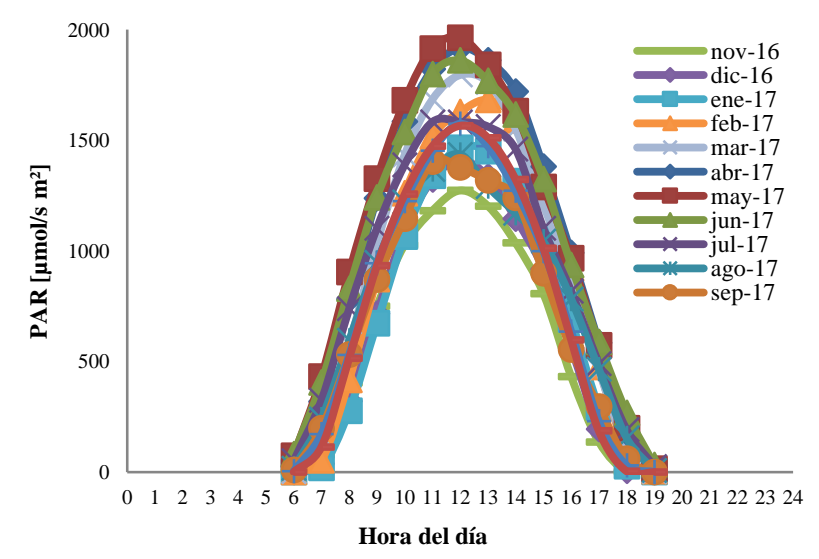

Gráfico 3 Promedios horarios mensuales en el periodo evaluado de noviembre de 2016 a noviembre de 2017

La radiación PAR promedio anual fue de $819.52 \mu \mathrm{mol}$ de fotones $\mathrm{m}^{-2} \mathrm{~s}^{-1}\left(179.47 \mathrm{Wm}^{-2}\right)$, en el año 2017 se registró una radiación solar global promedio de $471.98 \mathrm{~W} \mathrm{~m}^{-2}$ en la misma estación, es decir, la radiación PAR representó el 39\% de la radiación solar del año 2017.
De acuerdo con la información que presentan Sager et al., (1997), en lo que respecta a algunas plantas y sus respectivos requerimientos para su propagación, vegetación, afloramiento y el desarrollo de frutos o semillas, la cantidad de la PAR es idónea. Sólo hay que considerar los demás parámetros importantes para el crecimiento de las plantas, tales como, el fotoperiodo, nutrientes, $\mathrm{pH}$ y la temperatura.

\section{Conclusiones}

La radiación PAR promedio anual fue de 819.52 $\mu$ mol de fotones $\mathrm{m}^{-2} \mathrm{~s}^{-1}\left(179.47 \mathrm{Wm}^{-2}\right)$ en el año 2017 se registró una radiación solar global de $471.98 \mathrm{~W} \mathrm{~m}^{-2}$ en la misma estación, es decir la radiación PAR representó el 39 \% de la radiación solar del año 2017 un porcentaje dentro del rango esperado entre el $35 \%$ y el $50 \%$.

La radiación PAR necesaria para el desarrollo de cultivos y plantas también está relacionada ampliamente con el tipo de planta, ubicación geográfica, las características del lugar, y muchos otros factores; el promedio mensual promedio para cada mes presenta valores de radiación PAR desde el orden de los $590 \mu \mathrm{mol}$ de fotones $\mathrm{m}^{-2} \mathrm{~s}^{-1} 1$ a los $1020 \mu \mathrm{mol} \mathrm{de}$ fotones $\mathrm{m}^{-2} \mathrm{~s}^{-1}$ por lo tanto, por éste parámetro se considera una región para cultivos con requerimientos altos de radiación tales como la manzana, cereza, maíz, uva, olivo, durazno, pera, tomate y trigo, de acuerdo con el parámetro de radiación PAR necesario para estas plantas; sin embargo el desarrollo adecuado de los cultivos aquí mencionado está bajo otra serie de condiciones que tendrían que ser analizadas como es la concentración de nutrientes que necesarios, humedad, $\mathrm{pH}$, fotoperiodo y la temperatura.

\section{Referencias}

Abal, G. y Durañona, V. (2013). Manual técnico de Energía Solar Térmica Vol I: Fundamentos. Publicación electrónica Facultad de Ingeniería, UDELAR, Uruguay, Disponible en: http://www.energiasolar.gub.uy/documentos/ca pacitacion/manual_tecnico_solar_termica.pdf

Alados-Arboledas L., Olmo F.J., Alados I., Perez M. (2000) Parametric models to estimate photosyntetically active radiation in Spain. Agricultural and Forest Meteorology, 101; 187201. 
Alvarado, R. E. R., Garay, M. J. R., Estrada, Drouaillet B., Martínez, G. J. C., Rojas, G. A. R., Joaquín, C. S., (2020), Variación morfológica en Moringa oleifera Lam. a diferentes densidades de población. Revista Mexicana de Ciencias Agrícolas publicación especial número 2415 de abril - 30 de mayo, pp 165-176.

Bidwell, R. (1993) Fisiología Vegetal. A. G. T. Editor S. A. México, D. F.

Barceló, J., Nicolás, G., Sabater, B. y Sánchez R. (1987). Fisiología Vegetal $4^{\circ}$ Ed. Ediciones Píramide, S. A. Madrid, España.

Ciencia.nasa.gov (2019) obtenido de: https:/ciencia.nasa.gov/science-at-

nasa/2010/05feb_sdo

De Las Rivas, J. (2000). La luz y el aparato fotosintético. En: Fundamentos de de la fisiología vegetal. Azcón-Bieto, J. y Talón M. (Coords.) $2^{\circ}$ Ed.Edicions Universitat de Barcelona. Barcelona, España.

Durán E., Ángel Y. y Suárez J. (2015) Dinámica de la radiación fotosintéticamente activa en arreglos agroforestales con Hevea brasiliensis en el norte de la Amazona colombiana. Revista UDCA Actualidad \& Divulgación Científica 18 (2), 365-372.

Becker, P. and D. S. Weingarten. (1991). A comparison of several models for separating direct and diffuse components of solar irradiation.Agricultural and Forest Meteorology, 53; 347-353.

Benavides, H., Simbaqueva O. y Zapata H. (2017). Atlas de radiación solar U.V. y Ozono de Colombia. IDEAM. Bogotá, Colombia.

Bonhomme, R. (1993). The solar irradiation: Characterization and distribution in the canopy. In: Varlet-Grancher, C. el ál (eds). Crop estructure and light microclimate; characterization and applications.INRA (Francia), pp. 17-28.

Brown, T. L., LeMay, H. E. y Bursten, B, E., (2004), Química, la Ciencia Central, 9 ed. Pearson Educación, México.
Caballinas, R. (2017). El recurso solar en: Octavio G., Pilatowsky I. (Coords.) Aplicaciones térmicas de la energía solar, $1^{\circ}$.Edición, I.E.R. de la U.N.A.M., México, D.F.

Duffie, John A., William A. Beckman, (2013). Solar Engineering of the Thermal Processes, John Wiley \& Sons, $4^{\circ}$ Edición

Geofisica.unam.mx. 2019 obtenido de: http://www.geofisica.unam.mx/solarimetrico/re d_solarimetrica.html

Grossi, H. (2005) Distribución Espacial de la Radiación Fotosintéticamente Activa en Argentina. Meteorológica. 29. 27-36.

INEGI 2018 obtenido de:http://cuentame.inegi.org.mx/monografias /informacion

/zac/territorio/clima.aspx?tema $=$ me $\&$ e $=32$

Licor.com (2018) Obtenido de licor.com: https://es.licor.com/env/products/light/quantum. html

Liu, L., Veblen, K. E., \& Monaco, T. A. (2020). Shrub size modulates resource heterogeneity in a sagebrush-steppe ecosystem. Western North American Naturalist, 80(1), 28-37

Palomo, M., Montero, J., López, M., y Navascues, B. (2020). La Red Radiométrica en banda ancha de la Agencia Estatal de Meteorología. Acta de las Jornadas Científicas de la Asociación Meteorológica Española, (30).

Planas, G. A. G., (2020). Evaluación del sistema de iluminación artificial en céspedes deportivos. Universidad de las Islas Baleares.

Resnick R., HallidayD. y. Krane K. S, (1997) Física, v 2 4a edición, CECSA, México

Righini R., Grossi Gallegos H. (2005) Análisis de la correlación entre la radiación fotosintéticamente activa y la radiación solar global en San Miguel, provincia de Buenos Aires. Avances en Energías Renovables y Medio Ambiente, Vol.9 
Rojas, E. (2015). La radiación PAR y su efecto en los índices de crecimiento, rendimiento y calidad del cultivo del tomate. (Tesis de Maestría en Ciencias en Agroplasticultuta) Centro de Investigación Aplicada CIQA Coahuila México.

Sager J. and J.C. McFariane, (1997). Radiation en: Langhans, R. W. and Tibbitts, T. W. (Coords.) Plant Growth Chamber Handbook, $1^{\circ}$. Edición, Iowa State University, Iowa, E.U.A.

SimaoPeng, Quingyun Du, Aiwen Lin, Bo Hu, Ke Xiao, Yuliang Xi, (2015) Observation and estimation of photosyntetically active radiation in Lhasha (Tibetan Plateau). Advances in space research, 55; 1604-16012.

Tomoko Akitsu, Kenlo Nishida Nasahara, Yasuo Hirose, Osamu Ijima, (2015) Quantum sensors for accurate an stable long-term photosyntetically active radiation observations. Agricultural and Forest Metereology 237-238; 171-183

Torres, A. P., Lopez, R. G., (s.f.) Medición de Luz Diaria Integrada en Invernaderos. Purdure Extension.

Yang, F. (2020), Mecanismo ecológico de la construcción de la fuente de luz del espectro del paisaje y la nutrición del fósforo vegetal. ARCHIVOS LATINO AMERICANOS DE NUTRICIÓN Órgano Oficial de la Sociedad Latinoamericana de Nutrición Vol. $70 \mathrm{~N}^{\circ} 1$ (2020) pp. 404-411.

Zhian Sun, Lian Hong, Liu Jingmiao, ShiGuoping, (2017) Estimation of photosyntetically active radiation using solar radiation in the UV-visible spectre band. Solar Energy, 153; 611-622. 